PSU-TH $/ 237$

December 2000

\title{
NLO parton showers and subtractive techniques $^{1,2}$
}

\author{
F. Hautmann \\ Department of Physics, Pennsylvania State University, University Park PA 16802
}

\begin{abstract}
Motivated by asking how to combine parton showers with nonleading QCD matrix elements, we discuss a subtractive technique based on gauge-invariant Wilsonline operators and how this can be used to treat the soft region.
\end{abstract}

For precision phenomenology at future high energy colliders, it will be valuable to construct Monte Carlo event generators in which next-to-leading-order (NLO) QCD corrections can be incorporated in parton shower algorithms. There is at present no systematic method for doing this. Procedures based on subtractive methods have recently been proposed [1-3]. An important step in the implementation of this program is to show how to decompose Feynman graphs into sums of terms over different regions, with the terms arranged so as to correspond to factors in a factorization formula suitable for the Monte Carlo application.

An example of such a decomposition is given in Ref. [2] for the photon-gluon fusion process in leptoproduction. In this case soft gluons do not enter at the leading power, so that leading regions do not overlap. To handle general cases, however, one needs to treat graphs with soft gluons and hence with overlapping leading regions.

Fully numerical [4] or semi-analytical [5] subtraction methods have been devised to calculate NLO quantities that are infrared safe. These methods are not directly applicable in event generators that simulate the fully exclusive structure of the hadronic final states, since here the quantities being computed are not infrared safe in perturbation theory. In particular, one cannot use a cancellation of soft gluon contributions between real and virtual graphs.

The technique we discuss in this talk is constructed so that the following properties are satisfied [3]:

1) Talk at the Linear Collider Workshop LCWS2000, Fermilab, 24-28 October 2000.

2) Work supported in part by the US Department of Energy. 
(a) The integrand for the hard scattering coefficient is to be an integrable function, even when the corrections are applied to a process that is not infrared safe.

(b) The terms in the expansion of each Feynman graph should arise from matrix elements of gauge-invariant operators.

(c) In particular, the necessary cut-offs on rapidity integrations should be defined gauge-invariantly. This involves the use of Wilson lines along non-lightlike directions [6].

(d) The evolution equations [7] with respect to these cut-offs should be simple, in the sense that there should be no power-law remainder terms.

In what follows we illustrate this technique using a simple example, one-gluon emission graphs in leptoproduction.

We consider a generic observable associated with the reaction $\gamma^{*}(q)+q(p) \rightarrow$ $g(k)+q\left(k^{\prime}\right)$. We denote this by $\sigma[\varphi]$, where $\varphi$ is a weight function that contains the definition of the particular observable under consideration as a function of the final states as well as nonperturbative parts of the cross section, including the parton density. We denote by $\alpha$ and $\beta$ the gluon's fractional lightcone momenta, $\alpha=k^{+} /\left(x P^{+}\right), \beta=k^{-} /\left(Q^{2} /\left(2 x P^{+}\right)\right)$(with $x$ the Bjorken variable, $Q^{2}$ the photon virtuality, $P^{+}$the hadron's plus momentum), and by $\phi$ the gluon's azimuthal angle. We represent $\sigma[\varphi]$ as

$$
\sigma[\varphi]=\int_{0}^{\infty} d \alpha \int_{0}^{\infty} d \beta \int_{0}^{2 \pi} \frac{d \phi}{2 \pi} J(x, \alpha, \beta) M(\alpha, \beta) \varphi\left(x, Q^{2}, \alpha, \beta, \phi\right) .
$$

Here $M$ is the next-to-leading-order matrix element. For the $\gamma^{*} q$ amplitude obtained by contracting the photon Lorentz indices with the projector corresponding to the structure function $F_{2}, M$ is of the form

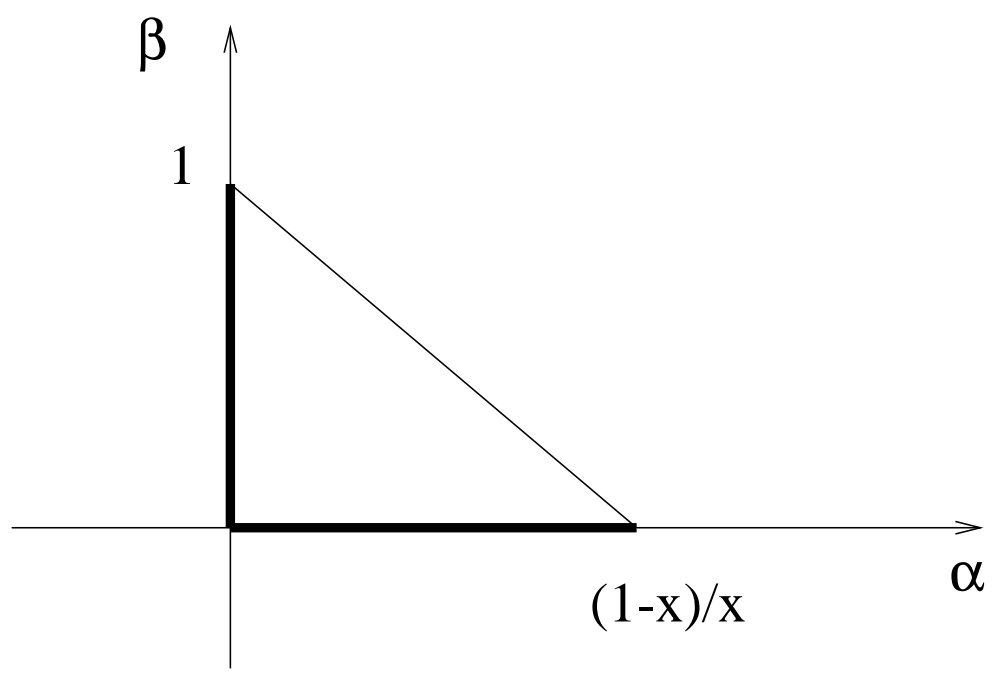

FIGURE 1. The phase space of Eq. (1) in the $\alpha, \beta$ plane. 


$$
M(\alpha, \beta)=(1-\beta)^{2} \frac{1+(1+\alpha-\beta)^{2}}{\alpha \beta(1+\alpha-\beta)}+2+6 \frac{(1-\beta)^{2}}{1+\alpha-\beta} .
$$

The factor $J$ in Eq. (1) is a Jacobian factor [3] and defines the physical region in the $\alpha, \beta$ plane as the interior of the triangle in Fig. 1 .

The infrared sensitive regions contributing to the leading power behavior of $\sigma[\varphi]$ are located in Fig. 1 as follows: the region in which the gluon is collinear to the initial state is a neighborhood of the axis $\beta=0$; the region in which the gluon is collinear to the final state is a neighborhood of the axis $\alpha=0$; the soft region is a neighborhood of the origin $\alpha=0, \beta=0$. The truly hard region lies away from the $\alpha=0$ and $\beta=0$ axes.

To obtain a decomposition for $\sigma$ into a sum of terms over the leading regions we employ the method of Ref. [6]. This generalizes the R-operation techniques of renormalization. See Ref. [8] for a related approach. We proceed from "smaller" to "larger" regions. For each region $R$, we remove the contribution from smaller regions, and construct an approximation to the matrix element valid in $R$, up to power suppressed corrections. Then we subtract any divergences that appear in this expression coming from larger regions. To ensure that the splitting between the terms is defined gauge-invariantly, at each step we demand that the counterterms be constructed from matrix elements of Wilson line operators,

$$
V_{I}(n)=\mathcal{P} \exp \left(i g \int_{-\infty}^{0} d y n \cdot A(y n)\right), \quad V_{F}(n)=\mathcal{P} \exp \left(i g \int_{0}^{+\infty} d y n \cdot A(y n)\right),
$$

with suitable directions $n$ for the lines. Evolution equations in $n$ enable one to connect the results corresponding to different directions. We introduce lightlike directions $\hat{p}=\left(1,0, \mathbf{0}_{T}\right), \hat{p}^{\prime}=\left(0,1, \mathbf{0}_{T}\right)$, and non-lightlike directions $u=$ $\left(u^{+}, u^{-}, \mathbf{0}_{T}\right), u^{\prime}=\left(u^{\prime+}, u^{\prime-}, \mathbf{0}_{T}\right)$ (all with positive components). We define $\eta=$ $\left(2 x^{2} P^{+^{2}} / Q^{2}\right) u^{-} / u^{+}$, and $\eta^{\prime}=\left(Q^{2} / 2 x^{2} P^{+^{2}}\right) u^{\prime+} / u^{\prime-}$.

The result for the term to be associated with the soft region reads [3]

$$
M_{S}(\alpha, \beta)=\frac{2}{\alpha \beta}-\frac{2}{\left(\alpha+\eta^{\prime} \beta\right) \beta}-\frac{2}{\alpha(\beta+\eta \alpha)} .
$$

The first piece in the right hand side is just the soft approximation $(\alpha, \beta \rightarrow 0)$ to Eq. (2). The other two pieces are the subtractions associated with the collinear regions where $\alpha / \beta$ or $\beta / \alpha$ go to zero. The first piece can be thought of as the oneloop contribution to the square of a vacuum-to-gluon matrix element of a product of eikonal Wilson lines taken along lightlike directions $\hat{p}, \hat{p}^{\prime}[6]$. The subtraction pieces can be derived from operators analogous to those for the first piece, except for replacing one of the lightlike eikonal lines by a line along a non-lightlike direction. The parameters $\eta, \eta^{\prime}$ act as gauge-invariant cut-off parameters. For example, in the second piece, which subtracts the divergence from the region $\beta / \alpha \rightarrow 0, \eta^{\prime}$ provides a cut-off on the region of small $\alpha$. 
By applying an analogous treatment we construct terms $M_{I}, M_{F}$ for the initialstate and final-state collinear regions. The term to be associated with the hard region is obtained by subtracting from the original matrix element the soft and collinear terms:

$$
M_{H}(\alpha, \beta)=M-M_{S}-M_{I}-M_{F}
$$

The explicit expression for $M_{H}$ is given in [3]. This expression is finite in all of the infrared regions, and independent of the choice of the non-lightlike directions $u, u^{\prime}$.

In the approach of $[2,3], M_{H}$ gives precisely the subtracted hard-scattering function to be used in the Monte Carlo to incorporate NLO corrections. The collinear terms correspond to factors in the showering. The soft term can be eliminated by a suitable choice of the directions for the Wilson lines: $M_{S}=0$ for $\eta \eta^{\prime}=1$. Then all of the infrared contributions are associated with either initial-state or final-state branchings, as is appropriate to match the structure of the parton shower.

It is interesting to consider the form of the collinear factors corresponding to the Monte Carlo scheme for the non-lightlike directions. In the symmetric case $\eta=\eta^{\prime}=1$, the result for the initial-state collinear factor is [3]

$$
M_{I}^{(\mathrm{MC})}(\alpha, \beta)=\frac{1}{\beta} \frac{1+(1+\alpha)^{2}}{\alpha(1+\alpha)}-\frac{1}{\alpha} \frac{2}{\alpha+\beta} .
$$

The first term in the right hand side corresponds to the standard form of the showering, with the coefficient of $1 / \beta$ given by the quark $\rightarrow$ quark splitting kernel. The second term, on the other hand, is non-standard. However, it is suppressed in the collinear region $\beta \rightarrow 0$ at fixed $\alpha$. That is, this form of the showering coincides with the standard one in the collinear limit and differs from it away from the collinear limit. The role of the second term is to provide an effective cut-off when $\alpha \rightarrow 0$, i.e., a cut-off on the soft singularity of the splitting kernel.

Observe that if one regulated the $\alpha \rightarrow 0$ behavior of the first term in Eq. (6) by subtracting its $\alpha \rightarrow 0$ limit, given by $2 /(\alpha \beta)$, this would bring about an extra $\beta \rightarrow 0$ singularity. This would not be suited for our application in a Monte Carlo algorithm. In contrast, the second term in Eq. (6) represents precisely what is, from our point of view, a better choice of a counterterm: it subtracts the $\alpha \rightarrow 0$ singularity without introducing any extra singular behavior at $\beta \rightarrow 0$.

Finally, note that this counterterm cuts off the integration over the region of small $\alpha$ at a value of order $\beta$. The procedure based on gauge-invariant subtractions tells us precisely where the cut-off is to be placed: the position of the cut-off on $\alpha$ turns out to be $\beta$-dependent. In more physical terms, this indicates that the cut-off to be applied in the initial-state shower and the cut-off to be applied in the final-state shower are not to be set independently, but they are related.

Acknowledgments. Thanks to J. Collins for collaboration on this topic. 


\section{REFERENCES}

1. C. Friberg and T. Sjöstrand, e-print hep-ph/9906316, in Proceedings of the DESY Workshop "Monte Carlo Generators for HERA Physics", eds. A.T. Doyle, G. Grindhammer, G. Ingelman and H. Jung (Hamburg, 1999), p.181.

2. J.C. Collins, JHEP 0005:004 (2000).

3. J.C. Collins and F. Hautmann, e-print hep-ph/0009286.

4. D.E. Soper, Phys. Rev. Lett. 81, 2638 (1998).

5. S. Catani and M.H. Seymour, Nucl. Phys. B485, 291 (1997); S. Frixione, Z. Kunszt and A. Signer, Nucl. Phys. B467, 399 (1996).

6. J.C. Collins and F. Hautmann, Phys. Lett. B 472, 129 (2000).

7. I.I. Balitsky and V.M. Braun, Nucl. Phys. B311, 541 (1989); J.C. Collins, in Perturbative Quantum Chromodynamics, ed. A.H. Mueller, World Scientific 1989, p. 573.

8. F.V. Tkachov, e-print hep-ph/9703423; Int. J. Mod. Phys. A8, 2047 (1993). 